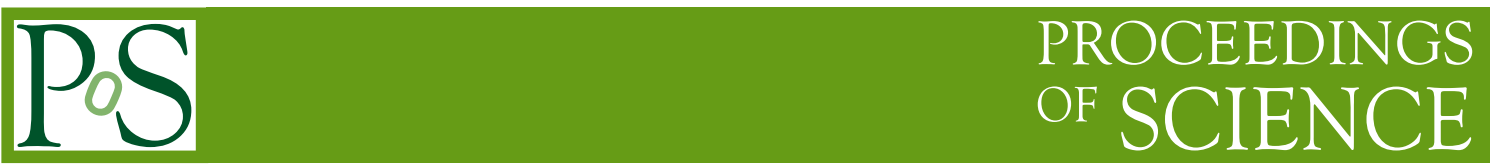

\title{
Nuclear forces in the parity odd sector and the LS forces
}

\author{
Keiko Murano* \\ RIKEN Nishina Center, RIKEN, Wako 351-0198, Japan \\ E-mail: murano@riken.jp
}

\section{for HAL QCD Collaboration}

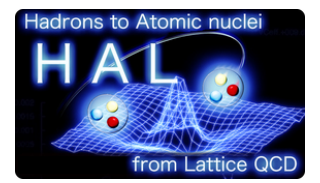

In this paper, we report our first attempt at determining NN potentials in the parity odd sector including the spin-orbit force in lattice QCD, employing the method to extract successfully parity even NN potentials from Nambu-Bethe-Salpeter (NBS) wave functions through the Schrödinger equation. Using $N_{f}=2$ CP-PACS gauge configurations on a $16^{3} \times 32$ lattice at $a \simeq 0.16 \mathrm{fm}$ and $m_{\pi} \simeq 1.1 \mathrm{GeV}$, we calculate central, tensor and spin-orbit potentials in the parity odd sector. Although statistical errors are still large, we observe that the qualitative features of these potentials roughly agree with those of phenomenological potentials.

The XXIX International Symposium on Lattice Field Theory - Lattice 2011

July 10-16, 2011

Squaw Valley, Lake Tahoe, California

\footnotetext{
* Speaker.
} 


\section{Introduction}

The nucleon-nucleon (NN) potential is one of the fundamental quantity to study various properties of atomic nuclei. In the past few decades, based on various models 1, 2] or the chiral effective field theory [4], several realistic NN potentials have been constructed to reproduce the $\mathrm{NN}$ scattering phases of more than 4000 data points with $\chi^{2} / N_{\text {dof }} \sim 1$. On the other hand, quite recently, a new method to extract NN potential in QCD has been proposed[5]. For the moment, the method has been successfully applied to the potentials in the parity even sector at the leading order of the derivative expansion, i.e., the central force $\left(V_{0}^{(+)}, V_{\sigma}^{(+)}\right)$and the tensor force $\left(V_{T}^{(+)}\right)$ are obtained for various cases including NN, YN, and YY systems [6 7 8] (where Y represents a hyperon) and meson-baryon system [9]. In addition, the method is recently extended to investigate $3 \mathrm{~N}$ forces 10. In contrast, the method has not yet applied to the forces in the parity odd sector as well as the spin-orbital force. They are needed for the complete determination of NN potentials at next-to-leading order (NLO). In particular, the spin-orbit force, even though it appears at NLO of a derivative expansion, is important, as is indicated by an analysis of phase shift[11, 12]. It is also important in explaining the ls-splitting of the (hyper) nuclear spectra and the magic numbers in nuclei, i.e., it induces the one-body spin-orbit term in the average single-particle nuclear potential[11, 13, 14].

The purpose of this paper is to extract potentials in the parity odd sector including the spinorbit force for the NN system. For this purpose, we construct NBS wave functions which include higher angular momenta, in order to determine the central force, the tensor force and the spin-orbit force in the parity odd sector $\left(V_{0}^{(-)}, V_{\sigma}^{(-)}, V_{T}^{(-)}, V_{L S}^{(-)}\right)$. Our calculation has been made by using $N f=2$ CP-PACS configurations on a $T \times L^{3}=16^{3} \times 32$ lattice at $\beta=1.95(a \simeq 0.16 \mathrm{fm})$ and $\kappa=0.1375\left(m_{\pi} \simeq 1100 \mathrm{MeV}\right)$.

\section{Extraction of potentials}

\subsection{Definition of potentials}

In the method, the NN potential is constructed form Nambu-Bethe-Salpeter (NBS) wave function in the center of mass (CM) frame, defined by

$$
\phi_{\alpha, \beta}(\mathbf{r} ; E) \equiv\left\langle 0\left|p_{\alpha}(\mathbf{x}) n_{\beta}(\mathbf{y})\right| p(+\mathbf{k}) n(-\mathbf{k})\right\rangle, \quad(\mathbf{r} \equiv \mathbf{x}-\mathbf{y})
$$

where $p_{\alpha}$ and $n_{\beta}$ denote local composite nucleon operators with spinor indices $\alpha, \beta$, and $E$ is kinetic energy related to the relative momentum $k=|\mathbf{k}|$ as $E=2 \sqrt{k^{2}+m_{N}^{2}}-2 m_{N}\left(\sim k^{2} / m_{N}\right)$ with $m_{N}$ being the nucleon mass. We define NN potentials from the NBS wave function below the inelastic threshold through the following Schrödinger type equation [15, 16]

$$
\left(E+\frac{\nabla^{2}}{m_{N}}\right) \phi(\vec{r} ; E)=\left[P^{+} V^{(+)}(r)+P^{-} V^{(-)}(r)\right] \phi(\vec{r} ; E)
$$

where $P^{+}\left(P^{-}\right)$denotes a projection operator for parity even (odd) with the corresponding potential $V^{(+)},\left(V^{(-)}\right)$, which is decomposed as

$$
V^{( \pm)}(r)=\left[V_{0}^{( \pm)}(r)+V_{\sigma}^{( \pm)}(r) \vec{\sigma}_{1} \cdot \vec{\sigma}_{2}+V_{T}^{( \pm)}(r) S_{12}+V_{L S}^{( \pm)}(r) \vec{L} \cdot \vec{S}+(\mathrm{NNLO})\right]
$$


with the central and spin-dependent central forces $V_{0}^{( \pm)}$and $V_{\sigma}^{( \pm)}$, the tensor force $V_{T}^{ \pm}$, and the spinorbit force $V_{L S}^{( \pm)}$. While $V_{0}^{( \pm)}, V_{\sigma}^{( \pm)}$and $V_{T}^{( \pm)}$are of leading order(LO) in the derivative expansion of non-local potentials, $V_{L S}^{ \pm}$appears at next-to-leading order(NLO). Once the above NBS wave functions are calculated in lattice QCD simulations, potentials can be extracted by solving eq. 2.2.

\subsection{Spin-singlet potentials}

For the spin-singlet and parity odd sector, the Schrödinger type equation eq.(2.2) is reduces to

$$
\left(E+\frac{\nabla^{2}}{m_{N}}\right) \phi_{S=0}^{(-)}(\vec{r} ; E)=V c_{S=0}^{(-)}(r) \phi_{S=0}^{(-)}(\vec{r} ; E),
$$

where $\phi_{S=0}^{(-)}(\vec{r} ; E)=P^{(-)} P^{S=0} \phi(\vec{r} ; E)$ with the spin-projection operator $P^{S}$ and $V c_{S=0}^{(-)}(r)=V_{0}^{-}(r)-$ $3 V_{\sigma}^{-}(r)$ denotes the central potential in the spin-singlet channel. From this equation, we then obtain

$$
V c_{S=0}^{(-)}(r)=E+\frac{1}{m_{N}} \frac{\nabla^{2} \phi(\mathbf{r} ; E)}{\phi(\mathbf{r} ; E)} .
$$

\subsection{Spin-triplet potentials including spin-orbit force}

For the spin-triplet and parity odd sector, the Schrödinger type equation eq.(2.2) reads

$$
\left(E+\frac{\nabla^{2}}{m_{N}}\right) \phi_{S=1}^{(-)}(\vec{r})=\left[V c_{S=1}^{(-)}(r)+V_{T}^{(-)}(r) S_{12}+V_{L S}^{(-)}(r) \vec{L} \cdot \vec{S}\right] \phi_{S=1}^{(-)}(\vec{r}), .
$$

Where $\phi_{S=1}^{(-)}(\mathbf{r})=P^{(-)} P^{S=1} \phi(\mathbf{r} ; E)$ and $V c_{S=1}^{(-)}(r)=V_{0}^{-}(r)+V_{\sigma}^{-}(r)$. Note that the isospin of these $V_{0}^{-}$and $V_{\sigma}^{-}$is different from that of the spin singlet sector.

In order to determine the spin-orbit potential for parity odd sector, we consider three linear independent set of NBS wave functions, $\phi^{X_{i}}$ with $i=1,2,3$, constructed by

$$
\phi^{X_{i}}(\vec{r})=P^{X_{i}} P^{S=1} P^{(-)} \phi(\vec{r} ; E) .
$$

where $P^{X_{i}}$ denotes a projection operator to state $X_{i}$, labeled by the orbital angular momentum $L$ and the total spin $J$. Eq. 2.6 is then decomposed into three independent equations as

$$
\left(E+\frac{\nabla^{2}}{m_{N}}\right) \phi^{X_{i}}(\vec{r})=P^{X_{i}}\left[V_{C}^{(-)}(r)+V_{T}^{(-)}(r) S_{12}+V_{L S}^{(-)}(r) \vec{L} \cdot \vec{S}\right] \phi(\vec{r}) .
$$

By solving these equations, potentials $V_{C}^{(-)}, V_{T}^{(-)}$and $V_{L S}^{(-)}$can be obtained as

$$
\left(\begin{array}{c}
V_{C}^{(-)}(r)-E \\
V_{T}^{(-)}(r) \\
V_{L S}^{(-)}(r)
\end{array}\right)=M(\vec{r})^{-1}\left(\begin{array}{c}
\nabla^{2} / m_{N} \phi^{X_{1}}(\vec{r}) \\
\nabla^{2} / m_{N} \phi^{X_{2}}(\vec{r}) \\
\nabla^{2} / m_{N} \phi^{X_{3}}(\vec{r})
\end{array}\right),
$$

where $M(\vec{r})$ denotes $3 \times 3$ matrix defined by

$$
M(\vec{r})=\left(\begin{array}{lll}
\phi^{X_{1}}(\vec{r}) & P^{X_{1}} S_{12} \phi(\vec{r}) & P^{X_{1}} \vec{L} \cdot \vec{S} \phi(\vec{r}) \\
\phi^{X_{2}}(\vec{r}) & P^{X_{2}} S_{12} \phi(\vec{r}) & P^{X_{2}} \vec{L} \cdot \vec{S} \phi(\vec{r}) \\
\phi^{X_{3}}(\vec{r}) & P^{X_{3}} S_{12} \phi(\vec{r}) & P^{X_{3}} \vec{L} \cdot \vec{S} \phi(\vec{r})
\end{array}\right) .
$$




\section{Construction of NBS wave functions in lattice QCD}

The NBS wave function is obtained from a 4-point nucleon correlation function on the lattice as

$$
\begin{aligned}
& G_{\alpha, \beta}\left(\vec{x}-\vec{y}, t-t_{0} ; \mathscr{J}\right) \equiv \frac{1}{L^{3}} \sum_{\mathbf{r}}\left\langle 0\left|T\left[p_{\alpha}(\vec{x}+\vec{r}, t) n_{\beta}(\vec{y}+\vec{r}, t) \mathscr{J}^{J=\tilde{J}, S=\tilde{S}}\left(t_{0}\right)\right]\right| 0\right\rangle \\
\simeq & \phi_{\alpha, \beta}^{J=\tilde{J}, S=\tilde{S}}\left(\vec{x}-\vec{y} ; E_{0}\right)\left\langle p\left(+\mathbf{k}_{0}\right) n\left(-\mathbf{k}_{0}\right)\left|\mathscr{J}^{J=\tilde{J}, S=\tilde{S}}(0)\right| 0\right\rangle e^{-E_{0}\left(t-t_{0}\right)}, \quad t-t_{0} \gg 1,
\end{aligned}
$$

where the summation over $\mathbf{r}$ is performed to select the two nucleon system with vanishing total spatial momentum. For $p(x)$ and $n(y)$, we employ the following local composite operators

$$
p(x) \equiv \varepsilon_{a b c}\left(u_{a}^{t}(x) \gamma_{5} d_{b}(x)\right) u_{c}(x), \quad n(x) \equiv \varepsilon_{a b c}\left(u_{a}^{t}(x) C \gamma_{5} d_{b}(x)\right) d_{c}(x),
$$

where $a, b$ and $c$ denote color indices, while $\mathscr{J}^{J=\tilde{J}, S=\tilde{S}}$ denotes a two-nucleon source operator with a definite total $\operatorname{spin} S=\tilde{S}(\tilde{S}=0,1)$ and a definite total "angular momentum" $J=\tilde{J}$, which is indeed an irreducible representation of the cubic group as $\tilde{J}=A_{1}, A_{2}, E, T_{1}, T_{2}$.

For such two-nucleon sources, we take two-nucleon momentum wall sources defined by

$$
\mathscr{J}_{\alpha \beta}(f) \equiv \bar{P}_{\alpha}(f) \bar{N}_{\beta}\left(f^{*}\right),
$$

where

$$
\begin{aligned}
& \bar{P}_{\alpha}(f) \equiv \sum_{\mathbf{x}_{1}, \mathbf{x}_{2}, \mathbf{x}_{3}} \varepsilon_{a b c}\left(\bar{u}_{a}\left(\mathbf{x}_{1}\right) C \gamma_{5} \bar{d}_{b}\left(\mathbf{x}_{2}\right)\right) \bar{u}_{c, \alpha}\left(\mathbf{x}_{3}\right) f\left(\mathbf{x}_{3}\right) \\
& \bar{N}_{\beta}(f) \equiv \sum_{\mathbf{x}_{1}, \mathbf{x}_{2}, \mathbf{x}_{3}} \varepsilon_{a b c}\left(\bar{u}_{a}\left(\mathbf{x}_{1}\right) C \gamma_{5} \bar{d}_{b}\left(\mathbf{x}_{2}\right)\right) \bar{d}_{c, \beta}\left(\mathbf{x}_{3}\right) f\left(\mathbf{x}_{3}\right)
\end{aligned}
$$

with $f$ being one of the following source functions, each of which corresponds to a plain wave parallel to one of spatial coordinate axes as

$$
\begin{array}{rlrl}
f^{(0)}(\mathbf{r}) & \equiv \exp (-2 \pi i x / L), & f^{(1)}(\mathbf{r}) \equiv \exp (-2 \pi i y / L), & f^{(2)}(\mathbf{r}) \equiv \exp (-2 \pi i z / L), \\
f^{(3)}(\mathbf{r}) \equiv \exp (+2 \pi i x / L), & f^{(4)}(\mathbf{r}) \equiv \exp (+2 \pi i y / L), & f^{(5)}(\mathbf{r}) \equiv \exp (+2 \pi i z / L) .
\end{array}
$$

Note that an element $g$ in the cubic group $O$ with 24 elements acts on these plane waves as

$$
f^{(i)} \mapsto \sum_{j} U_{i j}(g) f^{(j)},
$$

where the $6 \times 6$ representation matrix $U(g)$ are generated by representation matrices for $\mathbf{c}_{y}$ and $\mathbf{c}_{z}$, which are the rotations by 90 degree around $y$ and $z$ axes, respectively, and are explicitly given by

$$
U\left(\mathbf{c}_{y}\right) \equiv\left[\begin{array}{cccccc}
0 & 0 & 1 & 0 & 0 & 0 \\
0 & 1 & 0 & 0 & 0 & 0 \\
0 & 0 & 0 & 1 & 0 & 0 \\
0 & 0 & 0 & 0 & 0 & 1 \\
0 & 0 & 0 & 0 & 1 & 0 \\
1 & 0 & 0 & 0 & 0 & 0
\end{array}\right], \quad U\left(\mathbf{c}_{z}\right) \equiv\left[\begin{array}{llllll}
0 & 0 & 0 & 0 & 1 & 0 \\
1 & 0 & 0 & 0 & 0 & 0 \\
0 & 0 & 1 & 0 & 0 & 0 \\
0 & 1 & 0 & 0 & 0 & 0 \\
0 & 0 & 0 & 1 & 0 & 0 \\
0 & 0 & 0 & 0 & 0 & 1
\end{array}\right] .
$$


Note also that the spatial reflection for these source functions is represented by the complex conjugation. By a cubic group analysis, the orbital part of this momentum wall source is decomposed into $A_{1}^{+} \oplus E^{+} \oplus T_{1}^{-}$. Therefore, for the parity odd sector, $J^{P}$ we can access is $\left(L=T_{1}^{-}\right) \otimes\left(S=A_{1}\right)=T_{1}^{-}$ for the spin-singlet sector and $\left(L=T_{1}^{-}\right) \otimes\left(S=T_{1}\right)=A_{1}^{-} \oplus E^{-} \oplus T_{1}^{-} \oplus T_{2}^{-}$for the spin-triplet sector.

The wall source with the definite total angular momentum is now constructed as

$$
\mathscr{J}_{\alpha \beta}^{J=\tilde{J}}\left(f^{(i)}\right) \equiv \frac{d^{(\tilde{J})}}{24} \sum_{g \in O} \chi^{(\tilde{J})}\left(g^{-1}\right) U_{i j}(g) \mathscr{J}_{\alpha^{\prime} \beta^{\prime}}\left(f^{(j)}\right) S_{\alpha^{\prime} \alpha}^{-1}\left(g^{-1}\right) S_{\beta^{\prime} \beta}^{-1}\left(g^{-1}\right)
$$

where $d^{(\tilde{J})}$ and $\chi^{(\tilde{J})}(g)$ denote the dimension and the character for the irreducible representation $\tilde{J}$, respectively. Hereafter the Dirac indices $\alpha, \beta$ are restricted to upper components (in the Dirac representation). The total spin $S$ is projected by the spin projection operator $P^{(S)}$ as

$$
\mathscr{J}_{\alpha \beta}^{J=\tilde{J}, S=\tilde{S}}\left(f^{(i)}\right) \equiv P_{\alpha \beta, \gamma \delta}^{(\tilde{S})} \mathscr{J}_{\gamma \delta}^{J=\tilde{J}}\left(f^{(i)}\right),
$$

$P^{(S=0)} \equiv\left(1-\sigma_{1} \cdot \sigma_{2}\right) / 4$ and $P^{(S=1)} \equiv\left(3+\sigma_{1} \cdot \sigma_{2}\right) / 4$. Finally, the parity projection is defined by

$$
P^{( \pm)} \cdot \mathscr{J}_{\alpha \beta}\left(f^{(i)}\right)=\frac{1}{2}\left(\mathscr{J}_{\alpha \beta}\left(f^{(i)}\right) \pm \mathscr{J}_{\alpha \beta}\left(f^{(i) *}\right)\right)
$$

Although the orbital part for our two-nucleon source operators in the parity odd system consists of $L=T_{1}$ only, other orbital components of the NBS wave function at the sink are induced through the effect of the tensor force in the case of the spin-triplet sector. In this paper, for simplicity, we consider only an $L=T_{1}$ component of the NBS wave function as

$$
\phi_{L=T_{1}}^{J=\tilde{J}, S=\tilde{S}}\left(\mathbf{r} ; E_{0}\right) \equiv \sum_{g \in O} \chi^{\left(T_{1}\right)}(g)^{*} \phi^{J=\tilde{J}, S=\tilde{S}}\left(R(g) \mathbf{r} ; E_{0}\right) .
$$

where $R(g)$ denotes the rotation matrix for a cubic group element $g$, which rotates only the orbital components of the NBS wave functions as $\mathbf{x} \mapsto \mathbf{x}^{\prime} \equiv R(g) \mathbf{x}$.

\section{Lattice setup}

Our calculation is performed on a set of $N_{f}=2$ dynamical QCD configurations generated by the CP-PACS Collaboration on a $16^{3} \times 32$ lattice[17], who employs the $O(a)$-improved Wilson quark action with $C_{S W}=1.53$ at $\kappa_{u d}=0.1375$ and the RG improved gauge action (Iwasaki action) at $\beta=1.95$. This set of parameters corresponds to the lattice spacing $a=0.1555 \mathrm{fm}$ and pion mass $m_{\pi}=1136 \mathrm{MeV}$ ( nucleon mass $m_{N}=2165 \mathrm{MeV}$ ). The 4-point nucleon correlation function eq. (3.1) is calculated with the periodic and the Dirichlet boundary conditions along the spatial and the temporal directions, respectively. The spatial momentum of each nucleon is discretized as $k_{i} \simeq 2 \pi n_{i} / L\left(n_{i} \in \mathbb{Z}\right)$ in the periodic BC. Since $k=0$ state is forbidden in the parity odd system, the ground state energy with parity odd system is not $E=0$ but $E \simeq(2 \pi / L)^{2} / m_{N}$. In our analysis, we thus employ a free value $E=(2 \pi / L)^{2} / m_{N}=115 \mathrm{MeV}$ for an energy $E$ in eq. (2.9). 

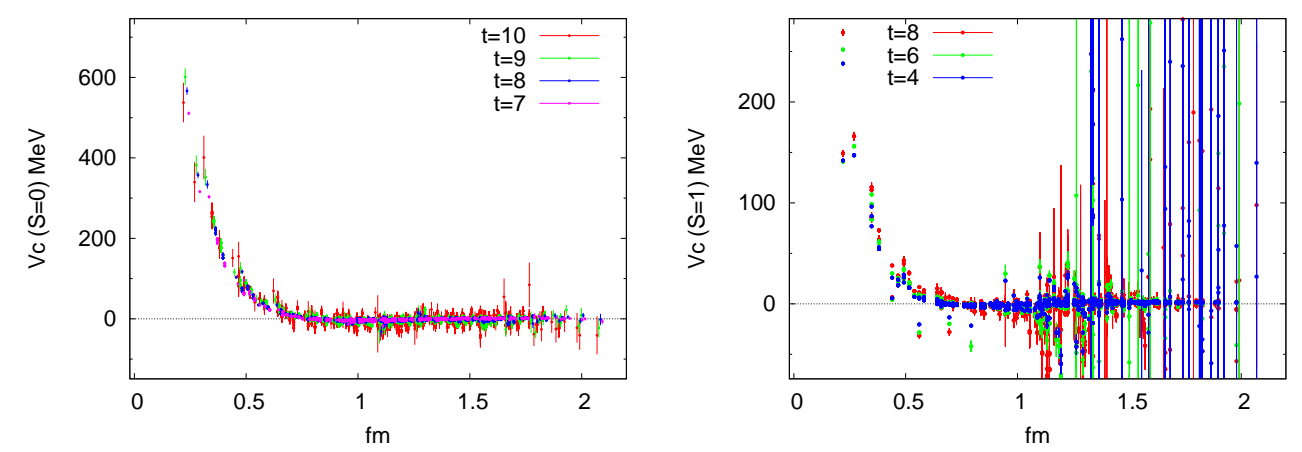

Figure 1: (Left) The central potential in the spin-singlet and parity odd sector, calculated from ${ }^{1} P_{1}$ NBS wave function. Data at $t-t_{0}=7,8,9,10$ are simultaneously plotted. (Right)The central potentials in the spintriplet and parity odd sector, calculated from ${ }^{3} P_{0},{ }^{3} P_{1}$ and ${ }^{3} P_{2}$ NBS wave functions. Data at $t-t_{0}=4,6,8$ are simultaneously plotted.

\section{Numerical results}

For the spin singlet sector, we calculate the $J^{P}=T_{1}^{-}$NBS wave function with $L=T_{1}, \phi_{L=T_{1}}^{J=T_{1}, S=0}$, whose dominant component corresponds to the ${ }^{1} P_{1}$ NBS wave function. Using eq. 2.5], we obtain the central potential in this channel. Preliminary results at $t-t_{0}=7-10$ are plotted in Fig. 1 (Left). We observe that the central potential has a strong repulsion at short distance $(r \leq 0.5 \mathrm{fm})$ without attraction at longer distance.

For the spin triplet sectors, we calculate three NBS wave functions $\phi_{L=\tilde{T} \tilde{T}_{1}}^{J=\tilde{J}=1}$ with $\tilde{J}=A_{1}, T_{1}$ and $E$, whose dominant components correspond to ${ }^{3} P_{0},{ }^{3} P_{1}$ and ${ }^{3} P_{2}$, respectively. Using these NBS wave functions, we extract spin-triplet potentials in parity odd sector. The central potential $V c_{S=1}^{(-)}(r)$ is shown in Fig. 10 (Right). Moreover the tensor potential $V_{T}^{-}(r)$ and the spin-orbit force $V_{L S}^{(-)}(r)$ are given in the left and right panels of Fig. 2, respectively. Large fluctuations at $r \simeq 1.2$ $\mathrm{fm}$ are caused by spatial boundaries at $L / 2=8$, which breaks the rotational symmetry, while large statistical errors of the tensor force in the parity odd sector may be mainly caused by the fact that its magnitude is significantly smaller (few MeV) than other potentials (few-hundred MeV). Although systematic and statistical uncertainties are still large in our preliminary results, we observe the following qualitative features. (1) $V_{C}$ is repulsive at all distance with a repulsive core at short distance. (2) $V_{T}$ is positive and quite small. (3) $V_{L S}$ is large and negative. These features qualitatively agree with those of phenomenological potentials [2].

\section{Conclusions}

In this paper, we have made a first attempt at determining NN potentials in the parity odd sector including the spin-orbit force. Using $N_{f}=2$ CP-PACS gauge configurations on a $16^{3} \times 32$ lattice at $a \simeq 0.16 \mathrm{fm}$ and $m_{\pi} \simeq 1100 \mathrm{MeV}$, we have reported our preliminary results on central forces, the tensor force and the spin-orbit force in the parity odd sector. Although statistical errors are still rather large, we have observed that the qualitative behaviors of these potentials roughly agree with those of phenomenological potentials. 

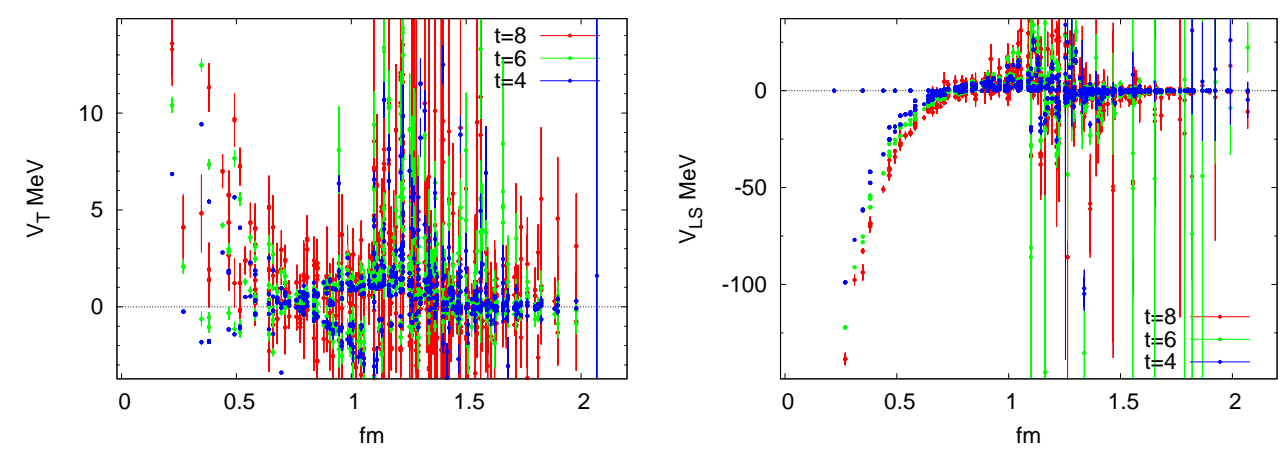

Figure 2: Potentials with spin-triplet state in parity odd sector, calculated from ${ }^{3} P_{0},{ }^{3} P_{1}$ and ${ }^{3} P_{2}$ NBS wave functions. Data at $t-t_{0}=4,6,8$ are simultaneously plotted. (Left) The tensor potential. (Right) The spinorbit potential.

Numerical calculations are performed on University of Tsukuba Supercomputer system (T2K). This work is supported by the Grant-in-Aid for Scientific Research on Innovative Areas(No.2004: 20105001, 20105003) and for Scientific Research(C) 23540321. We are grateful for authors and maintainer of CPS++[18] a modified version of which is used for this work.

\section{References}

[1] R. Machleidt, Phys. Rev. C 63, 024001 (2001).

[2] R. B. Wiringa, V. G. J. Stoks and R. Schiavilla, Phys. Rev. C 51, 38 (1995).

[3] V. G. J. Stoks, R. A. M. Klomp, C. P. F. Terheggen and J. J. de Swart, Phys. Rev. C 49, 2950 (1994).

[4] E. Epelbaum, H. -W. Hammer, U. -G. Meissner, Rev. Mod. Phys. 81, 1773-1825 (2009).

[5] N. Ishii, S. Aoki and T. Hatsuda, Phys. Rev. Lett. 99, 022001 (2007).

[6] H. Nemura, N. Ishii, S. Aoki and T. Hatsuda, Phys. Lett. B 673, 136 (2009).

[7] T. Inoue et al. [ HAL QCD Collaboration ], Phys. Rev. Lett. 106, 162002 (2011).

[8] T. Inoue et al. [HAL QCD Collaboration], Prog. Theor. Phys. 124, 591 (2010).

[9] Y. Ikeda [for HAL QCD Collaboration], arXiv:1111.2663 [hep-lat].

[10] T. Doi et al., arXiv:1106.2276 [hep-lat].

[11] A. Bohr and B. R. Mottelson, Nuclear Structure. (World Scientific Publishing Co., 1998). sect. 2-5.

[12] V. G. J. Stoks, R. A. M. Kompl, M. C. M. Rentmeester and J. J. de Swart, Phys. Rev. C 48, 792 (1993).

[13] M. G. Mayer, Phys. Rev. 75, 1969 (1949).

[14] J. H. D. Jensen and H. E. Suess, Phys. Rev. 75, 1766 (1949).

[15] S. Aoki, T. Hatsuda and N. Ishii, Prog. Theor. Phys. 123, 89 (2010).

[16] S. Aoki, T. Hatsuda and N. Ishii, Comput. Sci. Dis. 1, 015009 (2008).

[17] A. Ali Khan et al. [CP-PACS Collaboration], Phys. Rev. D 65, 054505 (2002) [Erratum-ibid. D 67, 059901 (2003)].

[18] Columbia Physics System (CPS), http://qcdoc.phys.columbia.edu/cps.html 